\title{
Preface to the Special Issue on “Frontier Researches of the High Temperature Properties of Slags"
}

\author{
Kazuhiro NAGATA \\ Department of Chemistry and Materials Science, Graduate School of Engineering, Tokyo Institute of Technology, 2-12-1, \\ Ookayama, Meguro-ku, Tokyo 152-8552 Japan.
}

The physical properties at the elevated temperatures are extremely important information for iron and steelmaker not only to improve the conventional processes but also to design new technologies. In spite of such importance, the number of available data in the literature and their accuracy are still limited due to the experimental difficulties at a high temperature. On the other hand, there have been great needs of precise physical properties of molten iron alloy, slags and fluxes in the iron- and steelmaking engineers. In order to meet such demands, the research group on "Physical properties of molten oxides at high temperature" (the leader, Professor Kazuhiro Nagata, Tokyo Institute of Technology) has been established in the Iron and Steel Institute of Japan (ISIJ) on 2002 as four years project. The main task of this group is collection, evaluation and selection of valuable data on the physical properties such as viscosity, interfacial/surface tension, thermal conductivity, refractive index and so forth for steelmaking slags and fluxes. The direct measurements of some properties have also been done by the members of this group. Furthermore, the modeling, estimation or prediction of the physical properties of slags as function of composition and temperature were attempted. The group consisted of three sub-groups; 1 . kinetic and structural properties (mainly viscosity), 2. surface/interfacial properties (mainly surface tension) and 3. thermal and optical properties (mainly heat conductivity) of slags, and the aimed slags in each sub-group are hot metal pre- treatment flux, BOF slags and mold powder for continuous casting, so that totally nine subjects have been investigated. In addition, some case studies to show how to use physical properties to simulate practical operation have also been introduced by this group according to the requests from steel industries as examples of application. Members of the research group have made significant contributions to such activities and the most of tasks of the group have been completed successfully.

As one of the research activities of the group, the special issue "Frontier Researches of the High Temperature Properties of Slags" has been organized. 17 excellent papers on viscosity, surface tension, thermal conductivity and refractive index of slags for the processes of continuous casting, pretreatment of hot metal and refining of steel have been contributed. These data were carefully examined in the research meetings of ISIJ and the international meeting on "Structure Models of Slags and Estimation of its Physical Properties" held in Sapporo city in autumn of 2003. These data will be separately published from ISIJ. We expect these data valuable for inventing new processes for iron and steelmaking. On behalf of the editors of this special issue, I would like to express my sincere appreciation to all the authors for their contributions and hope that this publication will be helpful for the engineers and researchers in this area. 